\title{
Periodic transient beam loading effect with passive harmonic cavities in electron storage rings
}

\author{
Tianlong He๑ ${ }^{*}$ Weiwei $\mathrm{Li}^{\dagger}{ }^{\dagger}$ Zhenghe Bai, ${ }^{\ddagger}$ and Lin Wang \\ National Synchrotron Radiation Laboratory, University of Science and Technology of China, \\ Hefei, Anhui, 230029, China
}

(Received 13 October 2021; accepted 18 January 2022; published 3 February 2022)

\begin{abstract}
In our previous study of bunch lengthening for the planned Hefei Advanced Light Facility (HALF) storage ring, a periodic transient beam loading effect was found in the presence of passive harmonic cavities for some cases, which can dramatically affect the bunch lengthening as well as the beam stability [T. L. He et al., Bunch lengthening of the HALF storage ring in the presence of passive harmonic cavities, in Proceedings of the IPAC2021, Campinas, SP (JACoW, Campinas, SP, Brazil, 2021), pp. 2082-2085, TUPAB265]. In this paper, we continue to use HALF as an example, via both tracking simulation and semianalytical calculation, to comprehensively study the characteristics of this periodic transient effect, analyze its dependence on some relevant factors and investigate the impact of fill pattern on its periodicity. It is found that there exits a threshold current for this periodic transient effect, which can be increased by the reduction of $R / Q$ of harmonic cavity (HC) and the increase of main cavity (MC) voltage, HC detuning, momentum compaction, and energy spread, as well as the inclusion of short-range wake. In addition, the periodicity of bunch centroid oscillation can be affected by the damping time and MC beam loading, and completely interrupted due to the nonuniform fill pattern, but this is not helpful for improvement of bunch lengthening.

DOI: 10.1103/PhysRevAccelBeams.25.024401
\end{abstract}

\section{INTRODUCTION}

The Hefei Advanced Light Facility (HALF) storage ring is designed toward a $2.2 \mathrm{GeV}$ diffraction-limited storage ring (DLSR) with natural emittance of $85 \mathrm{pm}$ rad and nominal beam current of $350 \mathrm{~mA}$ [1]. In order to suppress the emittance-diluting effects caused by intrabeam scattering effect and increase the Touschek lifetime, a passive superconducting 3rd-harmonic cavity (super-3HC) will be employed to lengthen the beam bunches. Assuming that the same super-3HC as the SLS/ELETTRA type [2] was used, we have recently studied, via tracking simulation and semianalytical calculation, the bunch lengthening driven by the fundamental mode of super-3HC for the HALF storage ring [3-5]. It was found that, in some cases, there existed a periodic dynamic solution, that is to say, the resulting rms lengths and centroid positions changed continuously and repeatedly with the number of tracking turns or numerical iterations. It was also found that this periodic dynamic effect could also be mitigated or even

\footnotetext{
*htlong@ustc.edu.cn

liwe@ustc.edu.cn

tbaizhe@ustc.edu.cn
}

Published by the American Physical Society under the terms of the Creative Commons Attribution 4.0 International license. Further distribution of this work must maintain attribution to the author(s) and the published article's title, journal citation, and DOI. eliminated by reducing $R / Q$ of harmonic cavity (HC) or increasing the main cavity (MC) voltage, both of which are usually adopted to reduce the static transient beam loading due to the asymmetric filling [6-8]. However, it should be emphasized that this periodic transient can especially happen in an uniform fill pattern and thus we call this new effect as the periodic transient beam loading effect (PTBL).

For a medium-low energy DLSR, in order to attain the ultralow emittance, it is necessary to lower the dispersion function at the arc sections, resulting in a small momentum compaction factor $\left(\sim 10^{-4}\right)$ [9]. Hence, the MC voltage can be reduced much for the same 5\% momentum acceptance. Meanwhile, the beam current should maintain the same level as that in the 3rd-generation light source. However, when facing the case of small momentum compaction factor, low MC voltage, and high beam current, the beam will become more sensitive to the disturbance due to $\mathrm{HC}$ beam loading, thereby increasing the possibility of PTBL. For example, it will be presented later that PTBL is predicted to occur at $260 \mathrm{~mA}$ for the HALF storage ring with bare lattice parameters. Besides HALF, the periodic dynamic solution for Advanced Light Source upgrade with distributed gaps was also obtained [5].

From the point of view of bunch motion, the typical feature of PTBL is that it is accompanied by periodic variations in bunch centroid positions and rms lengths, which is very similar to the instability with coupling between dipole and quadrupole Robinson modes as 
described in [10]. In addition, the bunch centroid distribution presents a coupled-bunch mode $l=1$, which is also similar to the instability described by Venturini with a perturbation-theory mode analysis [11]. However, it should be noted that there are significant differences between PTBL and the conventional coupled-mode or coupledbunch instabilities, although it shows a coupled-bunch motion and simultaneous oscillation of bunch centroid and length. One is that PTBL generally has a very long period, so it necessarily requires millions of tracking turns to be clearly observed. The other is that the occurrence of PTBL is independent of the radiation damping time setting, while the conventional coupled-bunch instability can be avoided if its growth rate is less than the radiation damping rate.

It is worth noting that stable (static) beam-loading transients with some of the bunches having double-bump profiles were once reported in $[8,12]$, which were observed by reducing the detuning of $\mathrm{HC}$ via measurements [12] and also simulations $[8,12]$. We found these results probably show PTBL. However, in view of that these transients were treated as "stable," it is believed that PTBL was not successfully revealed then.

In this paper, we will study the characteristics of PTBL in detail. The content of this paper is as follows. In Sec. II, we simply describe the characteristics of the tracking and semianalytical methods for studying PTBL, show that there is a threshold current for PTBL which can be determined by scanning the beam current, and prove that the results obtained by the two methods are in good agreement. In Sec. III, we illustrate the periodic characteristics of PTBL, including the periodic oscillation of centroid position and the periodic variation of bunch length, as well as the variation of centroid oscillation period and amplitude with the beam current. In Sec. IV, we introduce the dependence of threshold of PTBL on some relevant parameters or factors, such as MC voltage and $\mathrm{HC} R / Q$ (in Sec. IVA), $\mathrm{HC}$ detuning (in Sec. IV B), momentum compaction factor and energy spread (in Sec. IV C), radiation damping time (in Sec. IV D), short-range wake (in Sec. IV E) and MC beam loading (in Sec. IV F). In Secs. IV D-IV F, we also study their impacts on centroid oscillation. In Sec. V, we analyze the effect on PTBL due to the nonuniform fill pattern, including uniformly distributed gaps (in Sec. VA) and nonuniform charge configuration (in Sec. VB). Finally, we conclude our paper in Sec. VI.

\section{METHODS TO COMPUTE PTBL}

We have two methods to compute PTBL. One is the multiparticle tracking simulation with the usage of the STABLE code, which is implemented based on the GPUaccelerated algorithm and thus has high computational efficiency [5]. The other is the semianalytical calculation with a self-consistent code which solves the coupled Ha1ssinski equations iteratively $[4,13]$ and can be
TABLE I. Main parameters of the HALF lattice version A2 used for the following calculations.

\begin{tabular}{lc}
\hline \hline Parameter & Value \\
\hline Ring circumference & $480 \mathrm{~m}$ \\
Beam energy & $2.2 \mathrm{GeV}$ \\
Average beam current & $350 \mathrm{~mA}$ \\
Longitudinal damping time & $22.7 \mathrm{~ms}$ \\
Momentum compaction factor & $8.1 \times 10^{-5}$ \\
Natural energy spread & $6.45 \times 10^{-4}$ \\
Natural bunch length & $6.76 \mathrm{ps}$ \\
Harmonic number & 800 \\
Energy loss per turn & $198.8 \mathrm{keV}$ \\
Voltage of MC & $0.85 \mathrm{MV}$ \\
$R / Q$ of 3rd-HC & $90 \Omega$ \\
Quality factor of 3rd-HC & $5 \times 10^{5}$ \\
\hline \hline
\end{tabular}

accessible by the link [14]. Both of them can take into consideration beam loading effects in the main and harmonic cavities and short-range wakes for arbitrary fill patterns in electron storage rings. In this section, we would like to give a specific example to illustrate the characteristics of the two methods in calculating PTBL. The related calculation parameters of the HALF storage ring without insertion devices are summarized in Table I [1]. We first only consider the uniform and complete fill pattern and suppose that the only wakefield is from the HC. The HC detuning is determined by the near-optimum bunch lengthening condition [13].

Figure 1 shows the centroid position and $\mathrm{rms}$ bunch length over the turn number at beam current of 260 and $250 \mathrm{~mA}$ obtained by successive tracking simulation (The final bunch distributions of the case $260 \mathrm{~mA}$ will be taken as the input for the case $250 \mathrm{~mA}$ ). For the case of $250 \mathrm{~mA}$,

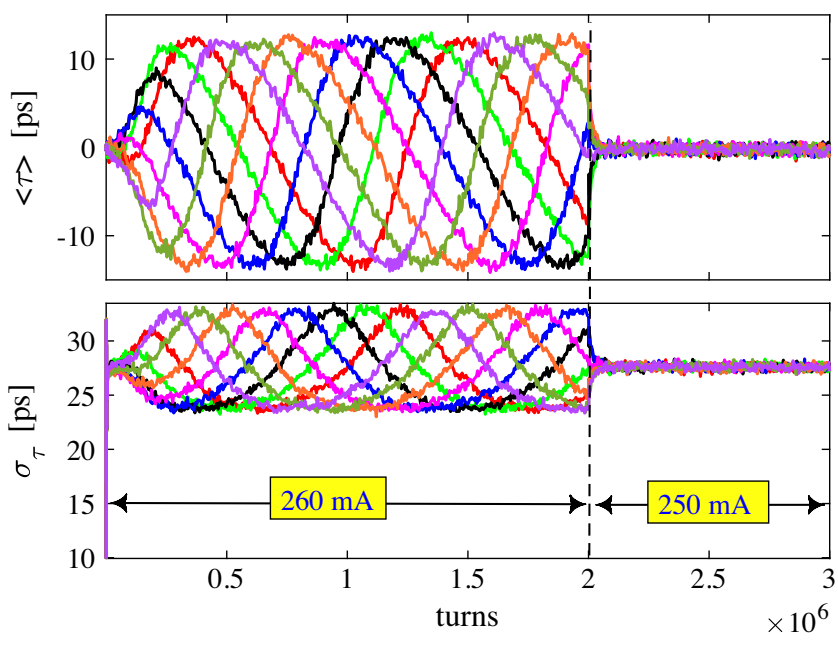

FIG. 1. Centroid positions (top) and rms bunch lengths (bottom) vs tracking turns, obtained with the STABLE code. Eight equally spaced bunches out of a total of 800 are displayed and distinguished by colors. 

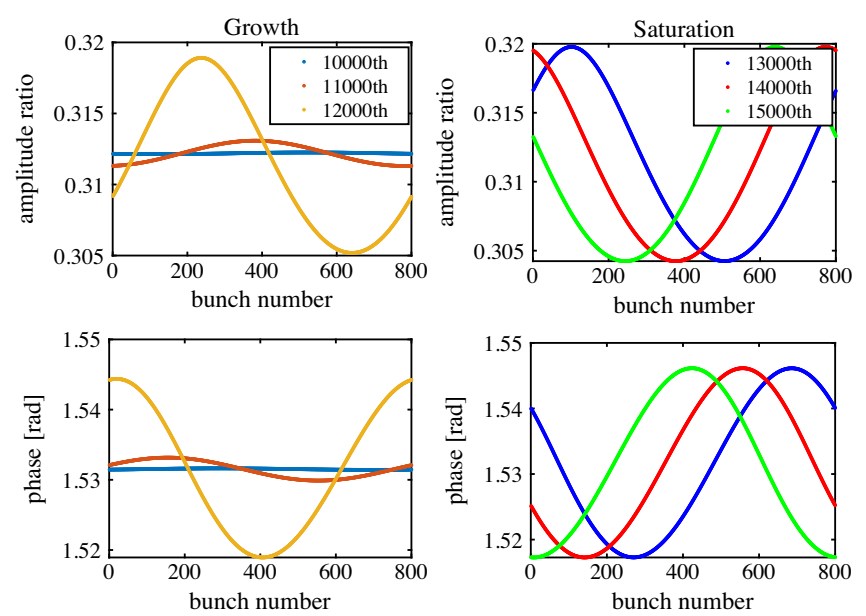

FIG. 2. HC voltage amplitude (top) and phase (bottom) vs bunch number at $260 \mathrm{~mA}$, given by the semianalytical calculation. The left column is the stage where the HC voltage difference continues to grow with the number of iterations, and the right column is the stage where saturation is reached. The legend gives the iteration number.

there exists a beam equilibrium with all bunches having the same profile excluding the impact of numerical errors. For the case of $260 \mathrm{~mA}$, we can see that both centroid position and rms bunch length of each bunch are finally oscillating periodically in the range of -14.2 to $13.0 \mathrm{ps}$ and 23.8 to $32.6 \mathrm{ps}$, respectively. The oscillation period can be estimated to $1.76 \mathrm{~s}$ based on the fact that it takes about $1.1 \mathrm{M}$ turns for one period and the revolution time is about $1.6 \mu \mathrm{s}$. It is clear that the threshold is just between 250 and $260 \mathrm{~mA}$, and can be further determined by taking smaller current range, but the precision is limited by the numerical noise. It indicates that the tracking method can be used not only to obtain the threshold, but also to determine the period length.

Figure 2 shows the voltage ratio and phase of $\mathrm{HC}$ obtained by the semianalytical calculation for the case of $260 \mathrm{~mA}$ (For the case of $250 \mathrm{~mA}$, the stable amplitude ratio and phase are 0.312 and 1.507 , respectively). Since it is slightly higher than the threshold current, we can see that the voltage difference between the bunches is tiny before $10 k$ th iterations, but continues to increase with the iteration steps. The difference becomes obvious after about $10 k$ iterations and reaches saturation at about $13 k$ iterations. Then the resulting distribution changes periodically with the number of iterations. According to calculation experience, the occurrence of the tiny but increasing difference during the iterative calculation process can be an indication for that the beam current is beyond the threshold, which can usually be observed within 200 iterations. In this way, the threshold can be efficiently determined by scanning the beam current.

Figure 3 shows the rms bunch lengths and centroid positions versus the bunch number obtained by the two
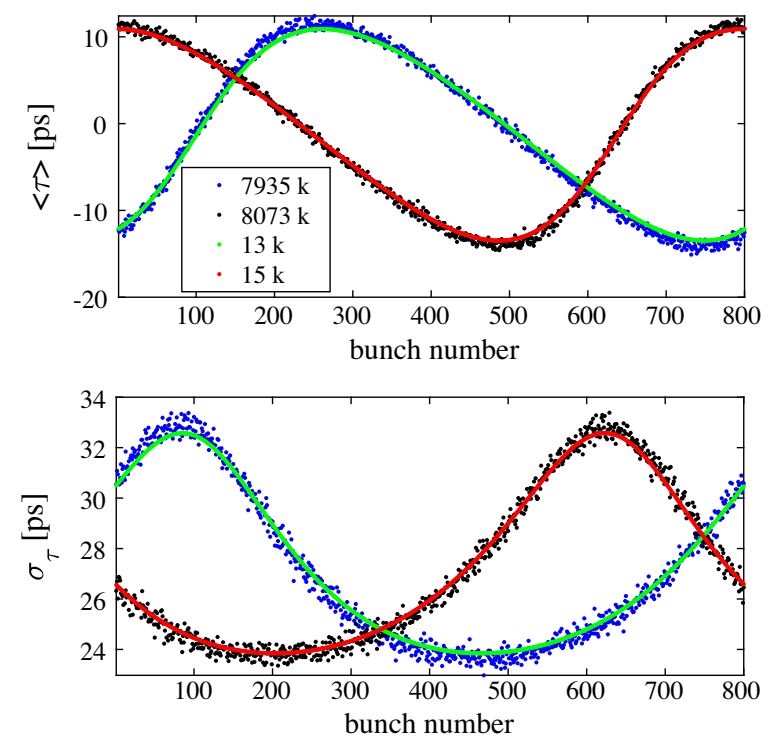

FIG. 3. Comparison between the tracking and semianalytical method. Centroid positions (top) and rms bunch lengths (bottom). The legend gives the tracking turn number (blue and black dots) and numerical iteration number (green and red dots).

methods. It can be seen that the final bunch distributions are in good agreement at some specific numbers of turns or iterations. So the semianalytical method could also be used to study PTBL, except that one cannot get the oscillation period since the iteration step is not directly related to any time step, but it is much faster compared with the tracking code.

It has been shown that the semianalytical method can be well used for studying PTBL. Nevertheless, for this typical nonequilibrium state, someone may be concerned about why this semianalytical method can work well for PTBL. As shown in Fig. 4, the semianalytical method mainly includes an iterative loop (see [13] for details). First, under the assumption of equilibrium state (unchanged bunch profiles) and given a bunch with density distribution $\rho_{i}(\tau)$ and charge $q_{i}$, the voltage phasor $\tilde{V}_{i, 0}$ induced by single pass of this bunch at $\mathrm{HC}$ is a complex phasor, which can be given as

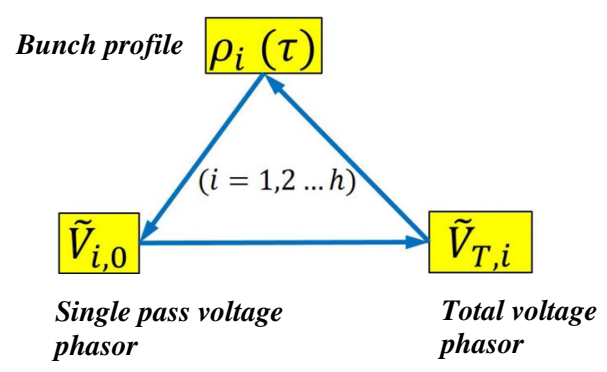

FIG. 4. Simplified diagram of the iterative scheme for bunch profiles by semianalytical method. 


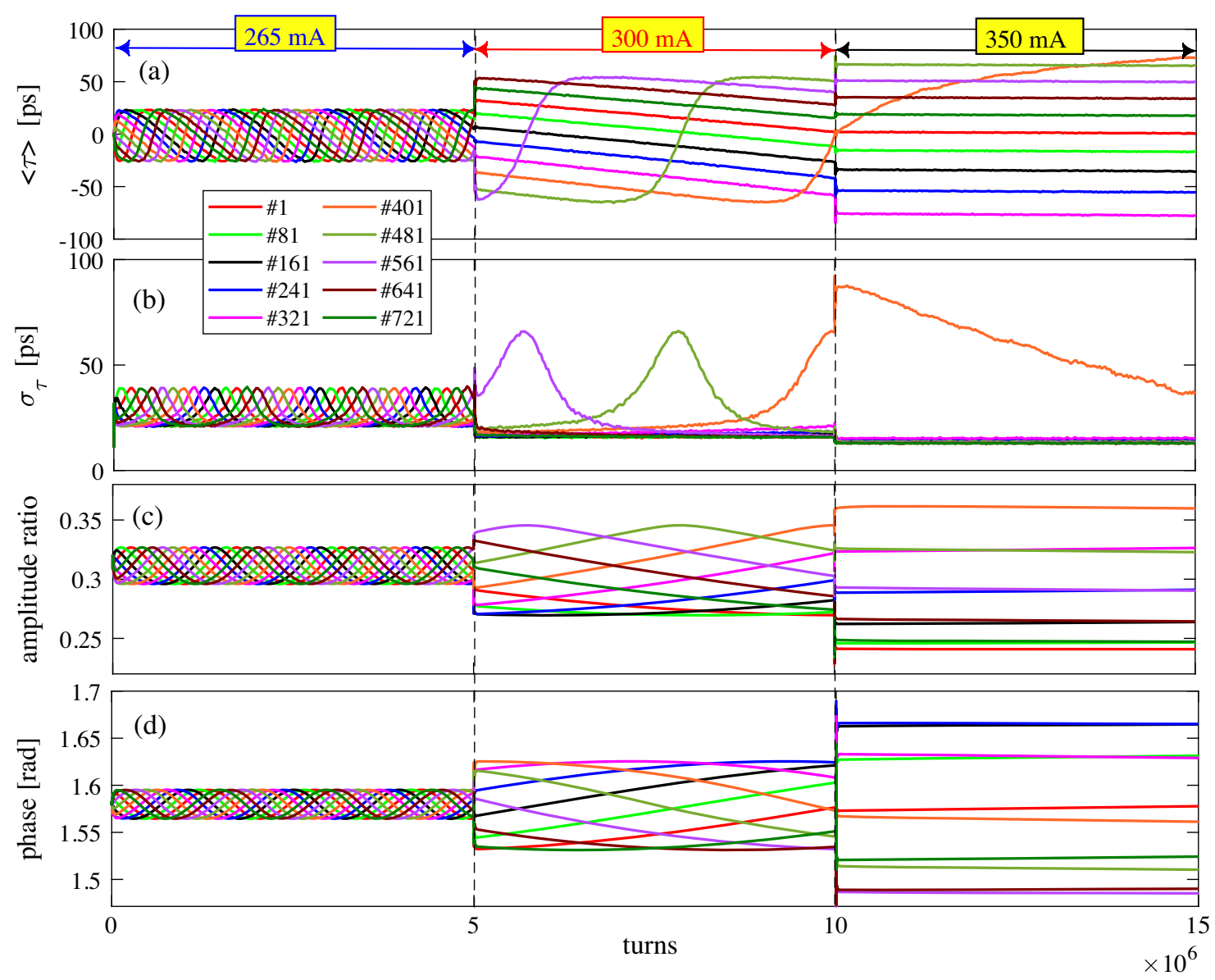

FIG. 5. Tracking results given by the STABLE code. (a) Centroid positions, (b) rms lengths, (c) HC voltage amplitude ratio, and (d) HC voltage phase. The legend gives the bunch number. The tracking simulation is successive for the three cases of 265,300 and $350 \mathrm{~mA}$. Ten equally spaced bunches out of a total of 800 are displayed and distinguished by colors.

$$
\tilde{V}_{i, 0}=\frac{q_{i} \omega_{r} R}{Q} \int_{-\infty}^{\infty} \rho_{i}(\tau) \exp \left[\left(-j+\frac{1}{2 Q}\right) \omega_{r} \tau\right] d \tau
$$

where $i=1,2, \ldots, h, \omega_{r}$ is the angular resonant frequency of $\mathrm{HC}$ and $j$ is the imaginary unit. Second, $\tilde{V}_{i, 0}$ can be used to obtain the $\mathrm{HC}$ voltage phasor $\tilde{V}_{H C, i}$ and thus the total voltage phasor $\tilde{V}_{T, i}$ seen by each bunch with the usage of the matrix method $[13,15]$. Third, the corresponding bunch density distribution $\rho_{i}(\tau)$ can be updated with $\tilde{V}_{T, i}$, so as to form an iterative loop.

It should be noted that this semianalytical method is based on the equilibrium assumption, which is not suitable for calculating the case of instability where the bunch profiles change rapidly. However, the period of PTBL is often very long, which means that the bunch profiles change slowly for many revolutions. It indicates that the assumption of equilibrium state is still approximately satisfied for PTBL when calculating the single pass voltage phasor $\tilde{V}_{i, 0}$ with Eq. (1). Therefore, the semianalytical method can also be used to calculate PTBL.

\section{PTBL CHARACTERISTICS}

As is known that an increase in beam current will exacerbate the static transient beam loading effect, which is also expected for PTBL. In this section, we will study the characteristics of PTBL dependent on beam current with the near-optimum bunch lengthening condition satisfied. Figure 5 shows the tracking results for three cases of 265 , 300 , and $350 \mathrm{~mA}$, which are obtained by successively tracking. The specific statistics are summarized in Table II with the inclusion of the case of $260 \mathrm{~mA}$. We can see that the oscillation ranges and periods of both centroid positions and rms lengths increase rapidly with beam current.

TABLE II. Statistics of the tracking results for PTBL.

\begin{tabular}{lccc}
\hline \hline $\begin{array}{l}\text { Current } \\
(\mathrm{mA})\end{array}$ & Period (s) & $\begin{array}{c}\text { Centroid } \\
\text { range }(\mathrm{ps})\end{array}$ & $\begin{array}{c}\text { RMS length } \\
\text { range }(\mathrm{ps})\end{array}$ \\
\hline 260 & 1.76 & -14.2 to 13.0 & 23.8 to 32.6 \\
265 & 2.35 & -26.5 to 24.0 & 20.5 to 39.3 \\
300 & 34.1 & -65.2 to 54.9 & 15.5 to 66.4 \\
350 & 977 & -93.9 to 78.5 & 12.7 to 89.4 \\
\hline \hline
\end{tabular}




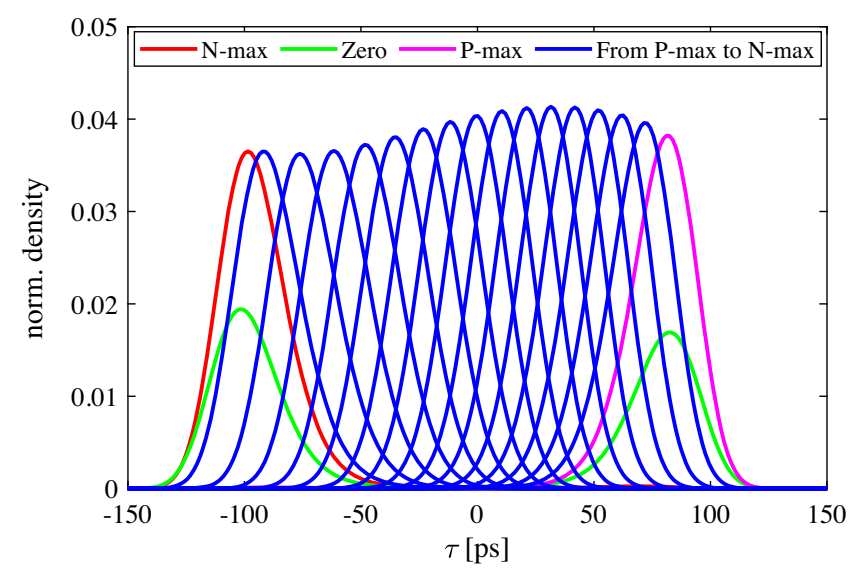

FIG. 6. Charge density profiles at different iteration steps for the case of $350 \mathrm{~mA}$, obtained by the semianalytical calculation. The legend gives the centroid positions. "N-max" and "P-max" represent the negative and positive maximum of centroid position, respectively. "Zero" denotes the zero position, and "From P$\max$ to N-max" means the centroid position moves from the positive maximum to the negative maximum.

Special attention should be paid to the case of $350 \mathrm{~mA}$ (the nominal current of HALF) where the tracking results of $5 M$ turns are displayed. It can be seen that in this time range, the centroid positions of most bunches seem to remain unchanged graphically, but in fact they are decreasing slowly and linearly. It should be noted that the decreasing range of centroid during the whole tracking can be used to estimate the period, which is too long to be directly obtained by tracking for the whole period. In addition, the rms lengths of most bunches are only about 13 ps which are slightly changed during the whole tracking, while individual bunches change significantly.

It can be seen that the variation of centroid position corresponds to that of rms length, which is more obvious for the case of $300 \mathrm{~mA}$ with bunch number 481 and 561that is, when the centroid moves rapidly from negative maximum to positive maximum, the bunch length first increases rapidly and reaches the peak (generally means a double-bump profile) at the zero centroid position, after that, it decreases rapidly. It is worth noting that the periodic variation of bunch length also means a periodic variation of bunch profile, which can be clearly seen in Fig. 6. Combined with that shown in Fig. 5, it is evident that the double-bump profile lasts for a relative short time during one period, especially when the beam current is much higher than the threshold current. Therefore, a heavy PTBL can contributes to a poor average bunch elongation.

It can be seen that PTBL is accompanied by the periodic variations of $\mathrm{HC}$ voltage phasor and bunch state. It hints that PTBL originates from the interplay between the two variations, which can drive and amplify each other until reaching saturation. This interplay is obvious because the two variations are linked according to the iterative loop shown in Fig. 4 and the tracking results shown in Fig. 5.
As is shown that PTBL can be enhanced via the increase of beam current (see Figs. 5), and it can be seen that the single pass voltage phasor $\tilde{V}_{i, 0}$ is linearly dependent on beam current. So it is reasonable to think that $\tilde{V}_{i, 0}$ plays an important role for PTBL. That is to say, PTBL is enhanced with higher $\tilde{V}_{i, 0}$, and vice versa, which can be easily checked by changing $\tilde{V}_{i, 0}$ and seeing what would happen (In fact, it has been preliminarily checked by changing the beam current, as shown in Fig. 5). According to Eq. (1), $\tilde{V}_{i, 0}$ can be reduced by the decrease of $q_{i} R / Q$, as well as the elongation of bunch. In the next section, it will be presented that the PTBL threshold current can be increased by reducing the $R / Q$-value and lengthening the bunch (caused by higher momentum compaction factor or higher relative energy spread or the short-range wake), and that PTBL will remain unchanged when $q_{i} R / Q$ and bunch profiles are held constant. These results make us believe that PTBL depends strongly on $\tilde{V}_{i, 0}$.

\section{DEPENDENCE OF THE PTBL THRESHOLD}

We have shown that the PTBL effect can cause undesirable bunch lengthening performance for the HALF storage ring at the nominal beam current of $350 \mathrm{~mA}$. To avoid its occurrence, there is a practical interest in exploring the dependence of the PTBL threshold on the relevant parameters or factors, including $\mathrm{MC}$ voltage, $\mathrm{HC} R / Q, \mathrm{HC}$ detuning, momentum compaction factor together with energy spread, longitudinal radiation damping time, as well as the effects of the short-range wake and MC beam loading (note that the momentum compaction factor depends on the specific lattice design and the radiation from damping wigglers will moderately increase the energy spread and reduce the damping time [16]). For the following calculations, it is assumed that the $r f$ buckets are filled completely with equal bunch charges, and that the HC detuning always satisfies the near-optimum lengthening condition unless otherwise specified. The threshold current can be obtained by current scan with the two aforementioned methods as presented in Sec. II. The semianalytical calculations agree well with the tracking simulations, which are much heavier computations and also much more noises, thus the semianalytical method will be used to quickly obtain the threshold and then the tracking threshold can be determined by scanning the beam current near the semianalytical threshold.

\section{A. MC voltage and $\mathrm{HC} R / Q$}

As is similar to the static transient beam loading effect related to the asymmetric filling pattern, PTBL is expected to be mitigated with higher MC voltage and lower $R / Q$. In this subsection, we will perform quantitative study on the relationship between the threshold of PTBL and this two factors. For HALF, in order to ensure 5\% momentum acceptance, the MC voltage shall be $0.85 \mathrm{MV}$. The $R / Q$ - 


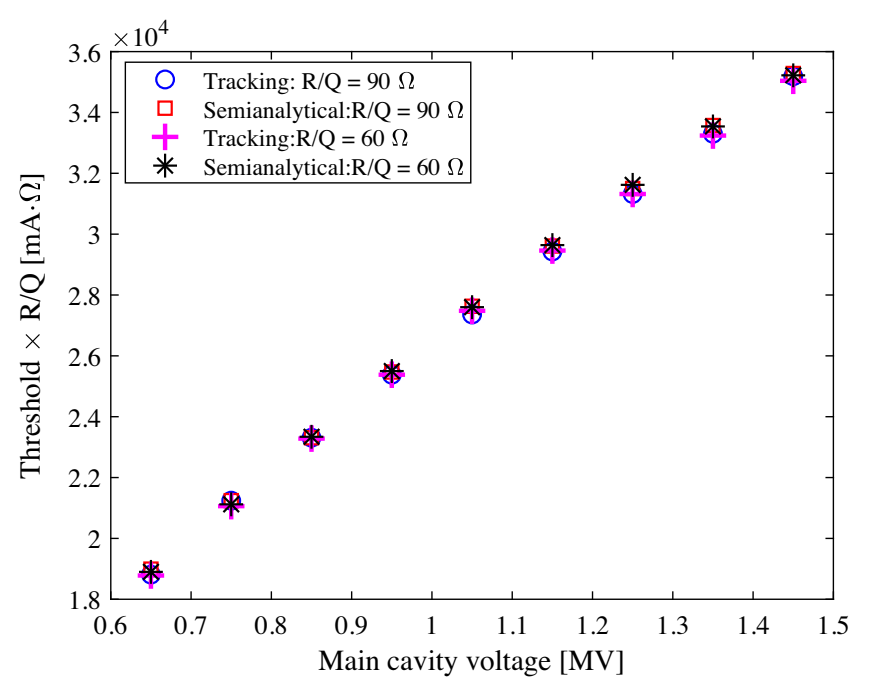

FIG. 7. The product of threshold current and $R / Q$-value as a function of the main cavity voltage.

value depends only on the cavity geometry, which can thus be reduced by optimizing the cavity shape [17]. The $R / Q$ of $\mathrm{HC}$ is first assumed to be $90 \Omega$, which is basically the same as that of SLS/ELETTRA type super-3HC (its maximum voltage is $1.0 \mathrm{MV}$ ). Since the required voltage of $\mathrm{HC}$ is about $0.27 \mathrm{MV}$ for HALF with bare lattice parameters, and it only reaches about $0.44 \mathrm{MV}$ even considering the extra energy loss from the insertion devices, therefore a $1.5 \mathrm{GHz} \mathrm{HC}$ with lower $R / Q$ is feasible for HALF, such as a superconducting cavity with only one cell. A second case with lower $R / Q$ of $60 \Omega$ is thus chosen to be studied.

For both aforementioned $R / Q$-values, we varied the MC voltage in the range of $0.65-1.45 \mathrm{MV}$ and calculated the corresponding threshold current by both aforementioned methods. The product of threshold current and $R / Q$-value as a function of the MC voltage is illustrated in Fig. 7. It is interesting to see that the results of four cases are well coincident, which means not only that the results obtained by the two methods agree well with each other, but also that the occurrence of PTBL actually depends on the product of $R / Q$-value and beam current for HALF with a specific MC voltage. Consequently, for a given $R / Q$-value, there is a threshold current. Conversely, for a given beam current, there is a threshold $R / Q$. The above relationship can help us quickly determine the threshold current corresponding to other $R / Q$-values, or determine the threshold $R / Q$ corresponding to a given beam current. For example, for the $\mathrm{MC}$ voltage of $0.85 \mathrm{MV}$, we have known that the threshold current related to the $R / Q$ of $90 \Omega$ is $259 \mathrm{~mA}$. In order to avoid PTBL under the nominal current of $350 \mathrm{~mA}$, the $R / Q$-value should be less than $66.6 \Omega$ without changing other parameters. This means that when the required current is high, the $R / Q$ should be reduced to a certain value.
It is worth noting that the results can be fitted into a straight line with a positive slope of about $20.4 \mathrm{~A} \Omega / \mathrm{MV}$. It indicates that the threshold current can be approximately linearly raised by the increase of the $\mathrm{MC}$ voltage, and can be more effectively raised with lower $R / Q$. However, we are not sure whether this approximately linear dependence of the threshold current on the main cavity voltage is still hold for other machine parameters, especially for that has normal conducting $\mathrm{HCs}$ with a significantly different resonant frequency or high total $R / Q$. More calculations are required to verify that.

\section{B. HC detuning}

The near-optimum lengthening condition fixes the corresponding relationship between beam current and $\mathrm{HC}$ detuning, while the change of the latter will cause the change of $\mathrm{HC}$ voltage and definitely has an impact on PTBL. Assuming that the fill pattern is uniform and there is no PTBL, the passive $\mathrm{HC}$ voltage excited by the circulating beam can be expressed as [18]

$$
V_{b}=-2 F I_{0} R \cos \left(\psi_{h}\right) \sin \left(n \varphi+\pi / 2-\psi_{h}\right),
$$

where $F$ is the bunch form factor which is about 1 for short bunches, $I_{0}$ is the average beam current, $R$ is the shunt impedance of $\mathrm{HC}$, and $\psi_{h}$ is the detuning angle defined in the range of 90 to $180 \mathrm{deg}$, which can be controlled by changing the detuning frequency according to the below formula

$$
\tan \left(\psi_{h}\right)=-\frac{2 Q \Delta f}{f_{r}},
$$

where $Q$ is the quality factor, and the $\mathrm{HC}$ detuning $\Delta f$ should be positive. So increasing the detuning frequency can increase the detuning angle (absolute value), and thus reduce the $\mathrm{HC}$ voltage amplitude. It is expected that its impact on the beam can also be reduced, so that the threshold current can be increased. As shown in Fig. 8, by adjusting the detuning to 19.2 and $34.57 \mathrm{kHz}$ for 350 and $500 \mathrm{~mA}$, respectively, the corresponding results separately become uniform, indicating that PTBL is completely suppressed. However, the corresponding rms length of each bunch is reduced to 18.5 and $12.2 \mathrm{ps}$, respectively, which illustrates that although this PTBL can be suppressed completely by increasing the detuning, it will lead to poor average bunch lengthening. And the higher the beam current, the poorer is the bunch lengthening.

\section{Momentum compaction factor and energy spread}

Besides the $r f$ parameters, the final bunch distributions are also closely related to the momentum compaction factor $\alpha_{c}$ and energy spread $\sigma_{\delta}$. To study their impact on the PTBL effect, we will change their values regardless of the ring lattice. 

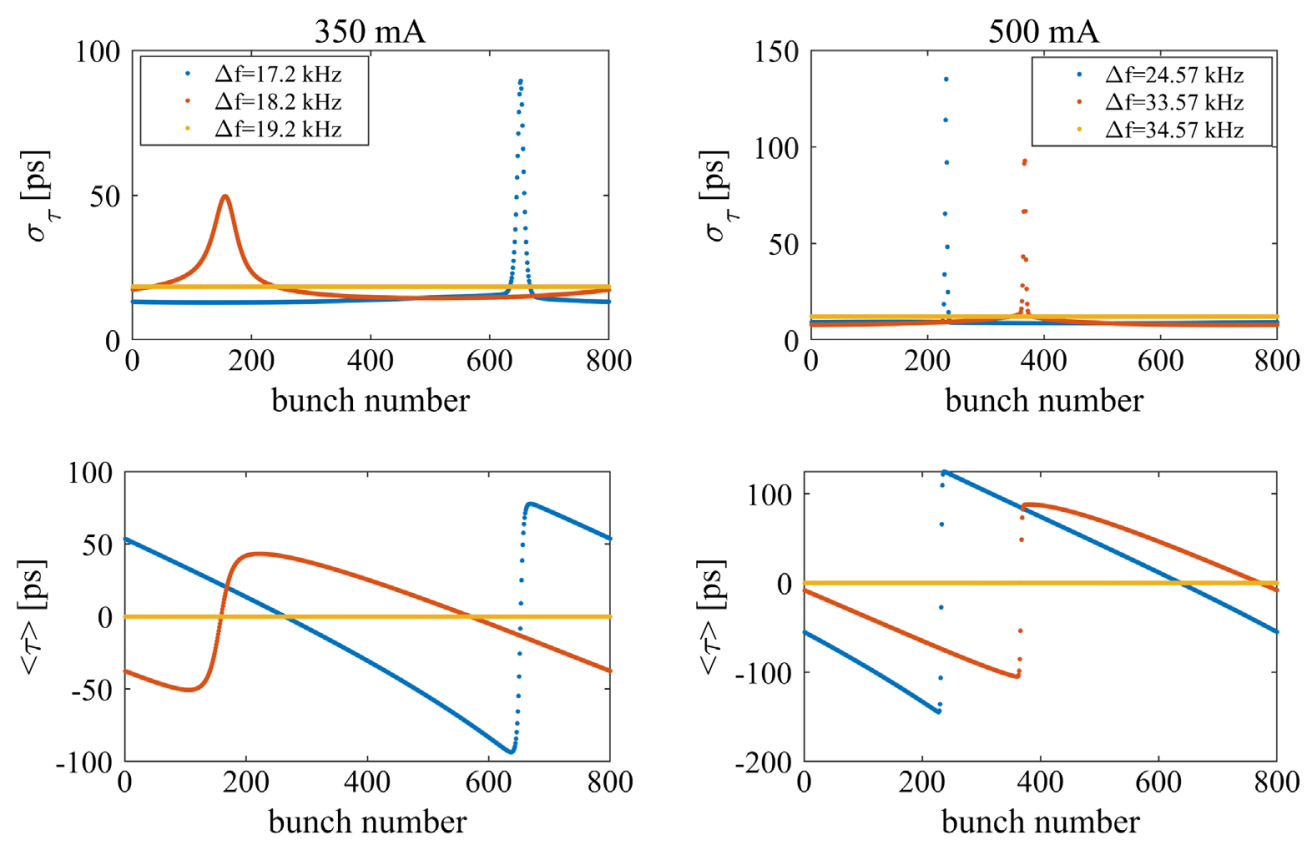

FIG. 8. RMS bunch lengths (top) and centroid positions (bottom) vs bunch number, obtained with the semianalytical method for the two cases of $350 \mathrm{~mA}$ (left column) and $500 \mathrm{~mA}$ (right column). The legend gives the detuning frequency, and $\Delta f=17.2 \mathrm{kHz}$ for the case of $350 \mathrm{~mA}$ is given by the near-optimum lengthening condition, as well as $\Delta f=24.57 \mathrm{kHz}$ for the case of $500 \mathrm{~mA}$.

For double $r f$ systems, the total longitudinal potential without the contribution from the short-range wake term can be given by [13]

$$
\begin{aligned}
\Phi(\tau)= & \frac{e V_{r f}}{2 \pi h \alpha_{c} E_{0}}\left\{\cos \left(\varphi_{s}\right)-\cos \left(\omega_{r f} \tau+\varphi_{s}\right)\right. \\
& \left.+\frac{k}{n}\left[\cos \left(\varphi_{h}\right)-\cos \left(n \omega_{r f} \tau+\varphi_{h}\right)\right]-\frac{U_{0} \omega_{r f} \tau}{e V_{r f}}\right\},
\end{aligned}
$$

where $V_{r f}, \omega_{r f}$, and $\varphi_{s}$ are the voltage amplitude, angular fundamental frequency, and synchronous phase of the main cavity, respectively, $k$ is the voltage ratio of the harmonic cavity relative to the main cavity, and $n$ and $\varphi_{h}$ are the harmonic order and synchronous phase of the harmonic cavity, respectively. For passive HC, $k$ and $\varphi_{h}$ are decided by the parameters of $\mathrm{HC}$ and bunch distributions. The bunch profiles can then be determined by the coupled Haissinski equations

$$
\rho(\tau)=\rho_{0} \exp \left[\frac{\Phi(\tau)}{\sigma_{\delta}^{2}}\right] .
$$

Thus the bunch density profile is a function of $\alpha_{c} \sigma_{\delta}^{2}$, so is the current threshold of PTBL. To verify this point, we can change the momentum compaction factor by a factor $X$ and the energy spread by the factor $X^{-1 / 2}$ while keeping all the other parameters in Table I unchanged. We have taken $X=1 / 3,1 / 2,2$, and 3 respectively. All the corresponding thresholds given by the semianalytical method remain to be $259 \mathrm{~mA}$. We have also studied the cases at the currents beyond the threshold with different $X$, and the resulting bunch distributions also keep almost the same.

We have also calculated the PTBL threshold with different momentum compaction factor or energy spread and the results are listed in Table III. It is clear that a greater value of $\alpha_{c} \sigma_{\delta}^{2}$ will result in a higher threshold current. One can better understand those results by considering the influence of the bunch shape on the beam loading voltage excited in the harmonic cavity. A greater value of $\alpha_{c} \sigma_{\delta}^{2}$ means a longer bunch with a smaller bunch form factor, which leads to the reduced single pass voltage phasor according to Eq. (1) with consequent decrease of HC beam loading voltage and thus the increase of PTBL threshold.

\section{Longitudinal radiation damping time}

We now discuss the impact of the damping time on PTBL with other parameters kept constant. As already studied in the above sections, the semianalytical method

TABLE III. Threshold affected by the momentum compaction factor $\alpha_{c}$ and energy spread $\sigma_{\delta}$, respectively. The results are obtained by the semianalytical method.

\begin{tabular}{lcccc}
\hline \hline \multicolumn{2}{l}{$\sigma_{\delta}=6.45 \times 10^{-4}$} & & \multicolumn{2}{c}{$\alpha_{c}=8.1 \times 10^{-5}$} \\
\cline { 1 - 2 } \cline { 5 - 5 }$\alpha_{c}\left(/ 10^{-5}\right)$ & Threshold $(\mathrm{mA})$ & & $\sigma_{\delta}\left(/ 10^{-4}\right)$ & Threshold $(\mathrm{mA})$ \\
\hline 6 & 240 & & 6.45 & 259 \\
8.1 & 259 & & 8 & 289 \\
10 & 271 & & 10 & 323 \\
20 & 322 & & 20 & 395 \\
\hline \hline
\end{tabular}



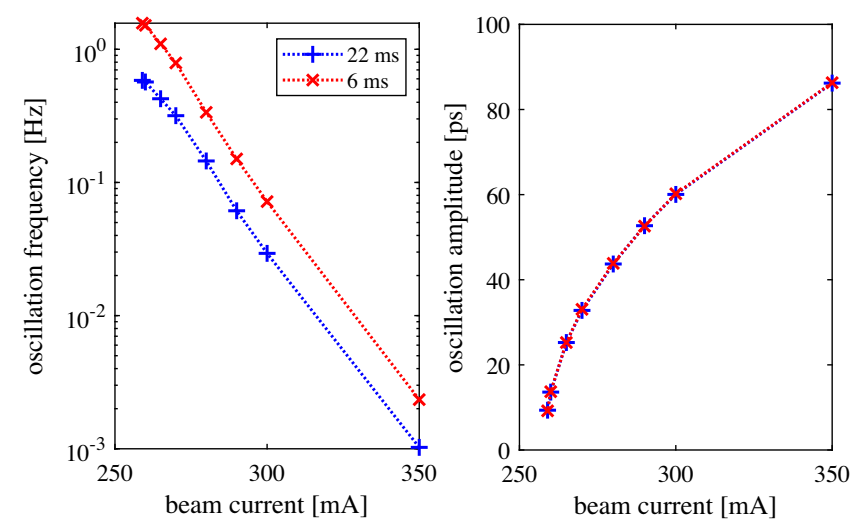

FIG. 9. Effect of the radiation damping time on PTBL. The oscillation frequency (left) is computed by taking the reciprocal of the oscillation period. The oscillation amplitude (right) is taken by half of the oscillation range.

can be used to give the PTBL threshold and bunch distributions, and good agreement is achieved in comparison with the tracking method. However, the semianalytical method does not contain the term of longitudinal damping time, thus the PTBL threshold is independent of the damping time. It should be noted that the damping time can affect the relative momentum deviation in each turn of the tracking method, which means it will change the tracking results. To illustrate its impact on the tracking results, Figure 9 shows the resulting oscillation frequencies and amplitudes at different currents with damping time of 22 and $6 \mathrm{~ms}$, respectively. The oscillation frequencies increase obviously and the amplitudes remain almost unchanged as the radiation damping time decreases from 22 to $6 \mathrm{~ms}$. Therefore a smaller radiation damping time will only speed up the process of PTBL.

\section{E. Short-range wake}

The longitudinal short-range wakes can cause bunch distortion and lengthening [19,20]. With the short-range wakefield taken into account, the PTBL threshold is expected to be increased as mentioned in the last paragraph of Sec. IV C. For simplicity, the short-range wake is represented by a single broad-band resonator model, with quality factor of 1 , resonant frequency of $30 \mathrm{GHz}$, and different values of shunt impedance. We can also study the PTBL effect by both semianalytical and tracking methods.

Still using the parameters listed in Table I and assuming no energy spread growth, the thresholds in the cases of the shunt impedance of 1,3 and $5 \mathrm{k} \Omega$ can be obtained by using the semianalytical method, which are 268, 287 and $299 \mathrm{~mA}$, respectively. It proves again that PTBL can be mitigated with a longer bunch. However, it should be noted that a strong short-range wake may cause bunch energy spread growth and thus the invalidation of the semianalytical method. So we should also use the tracking method to investigate the effect of the short-range wake on PTBL. In order to make the results more reliable, 50,000 macroparticles per bunch are used for tracking.

Figure 10 shows the oscillation frequency, oscillation amplitude and average of bunch energy spread as a function of beam current for six different cases, which are obtained after a large number of tracking turns until the variations across bunches become saturated. For the case of $1 \mathrm{k} \Omega$, the threshold is about $268 \mathrm{~mA}$, which is in good agreement with the semianalytical method since the energy spread has only a small increment. Apart from the increase of threshold, we can also see that the oscillation frequency increases by about 1 order of magnitude and the oscillation amplitude decreases obviously compared to the case of $0 \mathrm{k} \Omega$ (without short-range wake).
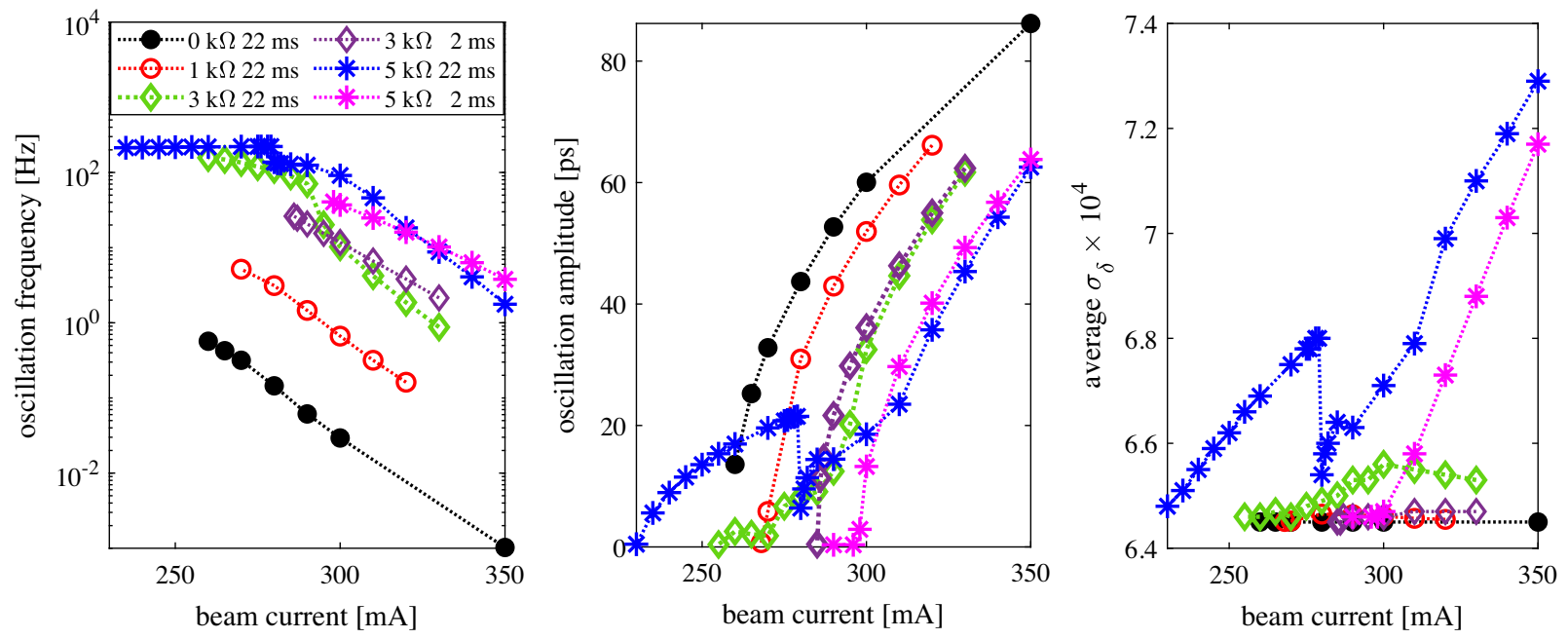

FIG. 10. Effect of the short-range wake on PTBL, obtained with the STABLE code. The legend gives the shunt impedance of the broadband resonator in $k \Omega$ and the radiation damping time in $\mathrm{ms}$. 
For the case of " $5 \mathrm{k} \Omega 22 \mathrm{~ms}$," the results become complex. We can see that the periodic phenomenon shows up at about $235 \mathrm{~mA}$, which is much lower than that given by the semianalytical method and even that given without the short-range wake. In the range of 235-279 mA, the oscillation frequency gradually rises from about 210 to $220 \mathrm{~Hz}$, which is significantly faster than that obtained without the short-range wake. Meanwhile, the bunch energy spread also oscillates periodically, and its amplitude increases with beam current. A similar situation also occurs in the case of " $3 \mathrm{k} \Omega 22 \mathrm{~ms}$," but its periodic motion appears at a higher current of about $260 \mathrm{~mA}$ with a slower frequency of about $157 \mathrm{~Hz}$, and the oscillation amplitude of bunch energy spread is obviously smaller than that of " $5 \mathrm{k} \Omega$ $22 \mathrm{~ms}$." However, if the damping time is reduced to $2 \mathrm{~ms}$, as the cases of " $5 \mathrm{k} \Omega 2 \mathrm{~ms}$ " and " $3 \mathrm{k} \Omega 2 \mathrm{~ms}$ " shown in Fig. 10, the energy spread can be effectively suppressed, and the thresholds are obtained as 297 and $285 \mathrm{~mA}$, respectively, which are consistent with those given by the semianalytical method.

According to the above results, it can be seen that when the short-range wake is strong to make the energy spread increase considerably, it may lead to a strong coupling between the short-range wake and the fundamental mode of HC. The typical phenomenon of this coupling is that the bunch energy spread also oscillates periodically. In addition, the centroid oscillation frequency is much faster than that in the case of without the short-range wake. We shall note that this coupling must exist, because there will be no periodic oscillation of bunch energy spread if only one of the two effects is considered.

We can also see that there is a sudden drop for the case of "5 k $222 \mathrm{~ms}$ " at $280 \mathrm{~mA}$. After that, the oscillation frequency decreases significantly as beam current increases, while the oscillation amplitude increases significantly, which is a typical characteristic of PTBL when without considering the short-range wake. It may indicate that the two wake effects are gradually decoupled as beam current increases above $280 \mathrm{~mA}$. However, it should be noted that this decoupling phenomenon is not well understood, which needs a further investigation.

To conclude, according to the results shown in Fig. 10, the presence of the short-range wake can significantly speed up the periodic oscillation, and the change of PTBL threshold depends upon the strength of the short-range wake. When it strongly causes a considerable increase in energy spread, there will appear a coupling between the short-range wake and the fundamental mode of $\mathrm{HC}$, resulting in the appearance of periodic oscillation of bunch energy spread, a much lower PTBL threshold and a much faster oscillation of bunch centroid.

\section{F. MC beam loading}

As is known for the complete fill case without PTBL, the beam loading voltage seen by each beam bunch will be the

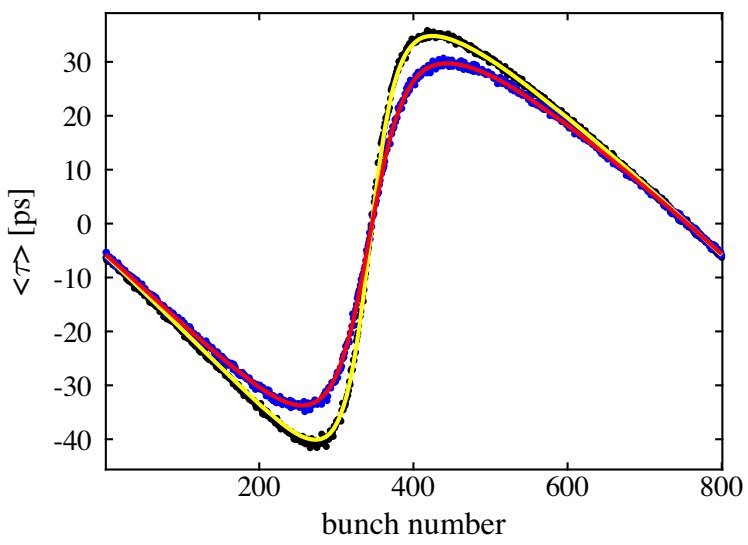

FIG. 11. Centroid position vs bunch number. The results of lines are obtained with the semianalytical method, while the dots with the STABLE code. The yellow line and black dots are corresponding to the case of with $\mathrm{MC}$ beam loading, and the red line and blue dots are for the case of without MC beam loading. Note that the resulting distributions have been shifted to coincide for easy comparison.

same. With an ideal beam loading compensation [14], the total MC voltage seen by each beam bunch is exactly the desired voltage. Therefore, it can be predicted that the MC beam loading will not affect the threshold current. However, when the current is beyond the threshold, the total $\mathrm{MC}$ voltage seen by each beam bunch will vary along the bunch number due to the fact that the beam loading compensation is for the average beam loading voltage. This variation of total MC voltage will affect the beam bunch motion. We next use the tracking and semianalytical methods to verify the above analysis. One provisional set of MC parameters are quality factor of $5.815 \times 10^{4}$, $R / Q$ of $90 \Omega$, and loading angle of about -10 degree controlled by shifting the detuning. By searching the threshold near $260 \mathrm{~mA}$, we finally obtained the same threshold of $258 \mathrm{~mA}$ via both methods. Then, we studied the case of $270 \mathrm{~mA}$, and the results of centroid positions with and without MC beam loading obtained by the two methods are shown in Fig. 11. Clearly, the MC beam loading can increase the centroid oscillation amplitude, for this case, by about 6 ps. In addition, the oscillation periods can also be estimated to be 4.9 (3.1) $\mathrm{s}$ with (without) $\mathrm{MC}$ beam loading. It indicates that the $\mathrm{MC}$ beam loading can also reduce the centroid oscillation frequency.

\section{FILL PATTERN}

The fill pattern usually refers to the configuration of empty $r f$ buckets (beam gaps) and filled bunch charges. In the preceding sections, we have studied PTBL with only the complete and uniform fill pattern. However there could be some reasons to introduce beam gaps in the fill pattern, such as ion clearing [21]. In addition, there is always charge 
TABLE IV. The state of results for the fill pattern with different cases of $N_{e} \times N_{p}$. I: identical subtrains; P: periodic variations; D: different subtrains.

\begin{tabular}{lcccccccccc}
\hline \hline Current $[\mathrm{mA}]$ & $1 \times 80$ & $2 \times 40$ & $4 \times 20$ & $5 \times 16$ & $8 \times 10$ & $10 \times 8$ & $16 \times 5$ & $20 \times 4$ & $40 \times 2$ & $80 \times 1$ \\
\hline 260 & $\mathrm{I}$ & $\mathrm{I}$ & $\mathrm{I}$ & $\mathrm{I}$ & $\mathrm{I}$ & $\mathrm{I}$ & $\mathrm{I}$ & $\mathrm{I}$ & $\mathrm{D}$ & $\mathrm{D}$ \\
265 & $\mathrm{P}$ & $\mathrm{P}$ & $\mathrm{P}$ & $\mathrm{P}$ & $\mathrm{P}$ & $\mathrm{P}$ & $\mathrm{D}$ & $\mathrm{D}$ & $\mathrm{D}$ & $\mathrm{D}$ \\
270 & $\mathrm{P}$ & $\mathrm{P}$ & $\mathrm{P}$ & $\mathrm{P}$ & $\mathrm{D}$ & $\mathrm{D}$ & $\mathrm{D}$ & $\mathrm{D}$ & $\mathrm{D}$ & $\mathrm{D}$ \\
300 & $\mathrm{P}$ & $\mathrm{P}$ & $\mathrm{D}$ & $\mathrm{D}$ & $\mathrm{D}$ & $\mathrm{D}$ & $\mathrm{D}$ & $\mathrm{D}$ & $\mathrm{D}$ & $\mathrm{D}$ \\
350 & $\mathrm{P}$ & $\mathrm{D}$ & $\mathrm{D}$ & $\mathrm{D}$ & $\mathrm{D}$ & $\mathrm{D}$ & $\mathrm{D}$ & $\mathrm{D}$ & $\mathrm{D}$ & $\mathrm{D}$ \\
\hline \hline
\end{tabular}

variation across bunches due to injection and lifetime issues. Obviously, fill gaps and charge variations will modulate the beam induced $\mathrm{HC}$ voltage and thus change the beam motion, resulting in a change of PTBL. For simplicity, we will study the two kind of nonuniform fill patterns separately and only consider the induced voltage from the fundamental mode of $\mathrm{HC}$.

\section{A. Uniformly distributed gaps}

We simply consider uniformly distributed gaps of $10 \%$ in the fill pattern and equal charge distributions for HALF. Thus the 800 buckets can be divided into $N_{p}$ periods, each with $N_{b}$ successive filled bunches and $N_{e}$ successive empty buckets, where $N_{p}, N_{b}$, and $N_{e}$ are positive integers with

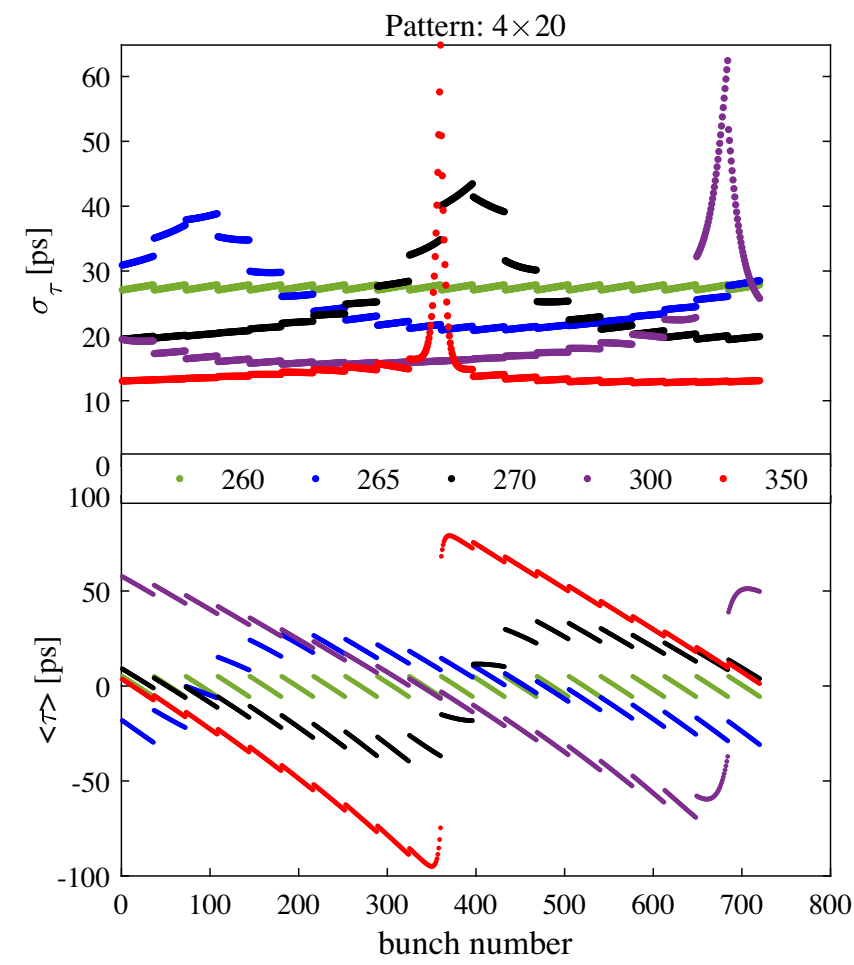

FIG. 12. RMS bunch length (top) and centroid position (bottom) vs bunch number, obtained by the semianalytical method. The legend gives the beam current in $\mathrm{mA}$. Note that for the cases of 265 and $270 \mathrm{~mA}$, the resulting distributions are periodically varied with the number of iterations, while only the results of the final iteration are plotted.
$N_{b} \times N_{p}=720$ and $N_{e} \times N_{p}=80$. It is easy to know that the value of $N_{p}$ can be 80, 40,20,16,10, 8, 5, 4, 2, and 1 . Note that the case of $N_{p}=1$ is special, since it means that there is only a single long gap following a singletrain beam.

We studied these cases using the semianalytical method since it is much faster. The current varies from 260 to $350 \mathrm{~mA}$. The results for different period numbers and currents can be classified into three types according to their final state, which are summarized in Table IV. In order to make these three types of results more clear, the results of the case of " $4 \times 20$ " were chosen to be illustrated in Fig. 12 . As presented in Table IV, the symbol " $I$ " means that the results are convergent and the bunch forms between the subtrains are identical (see the case of $260 \mathrm{~mA}$ shown in Fig. 12), the symbol " $P$ " means the resulting distribution changes periodically with the number of iterations, and the symbol " $D$ " means that the results are also convergent but the bunch forms between the subtrains are different (see the cases of 300 and $350 \mathrm{~mA}$ shown in Fig. 12). From Table IV and Fig. 12, it can be found that for the case of small gap width, the state of results shows a trend changed from " $I$ " to " $P$ " and then to " $D$ " as beam current increases. Meanwhile, no matter whether it is periodic or convergent the centroid transient becomes more severe with some of the bunches having double-bump profiles. We also found that the larger the gap width between two adjacent subtrains, the narrower the beam current range corresponding to $P$. This can be understood as that the state difference between two neighboring subtrains will grow with gap width and beam current, which can result in the termination of periodic motion if the state difference is too large.

TABLE V. The state of results for the complete fill pattern with different Gaussian random error. C: convergent; P: periodic variations.

\begin{tabular}{lccc}
\hline \hline Current $[\mathrm{mA}]$ & $1 \%$ & $2 \%$ & $5 \%$ \\
\hline 240 & $\mathrm{C}$ & $\mathrm{C}$ & $\mathrm{C}$ \\
250 & $\mathrm{C}$ & $\mathrm{C}$ & $\mathrm{C}$ \\
260 & $\mathrm{C}$ & $\mathrm{C}$ & $\mathrm{C}$ \\
270 & $\mathrm{P}$ & $\mathrm{C}$ & $\mathrm{C}$ \\
300 & $\mathrm{P}$ & $\mathrm{P}$ & $\mathrm{C}$ \\
350 & $\mathrm{P}$ & $\mathrm{P}$ & $\mathrm{C}$ \\
\hline \hline
\end{tabular}



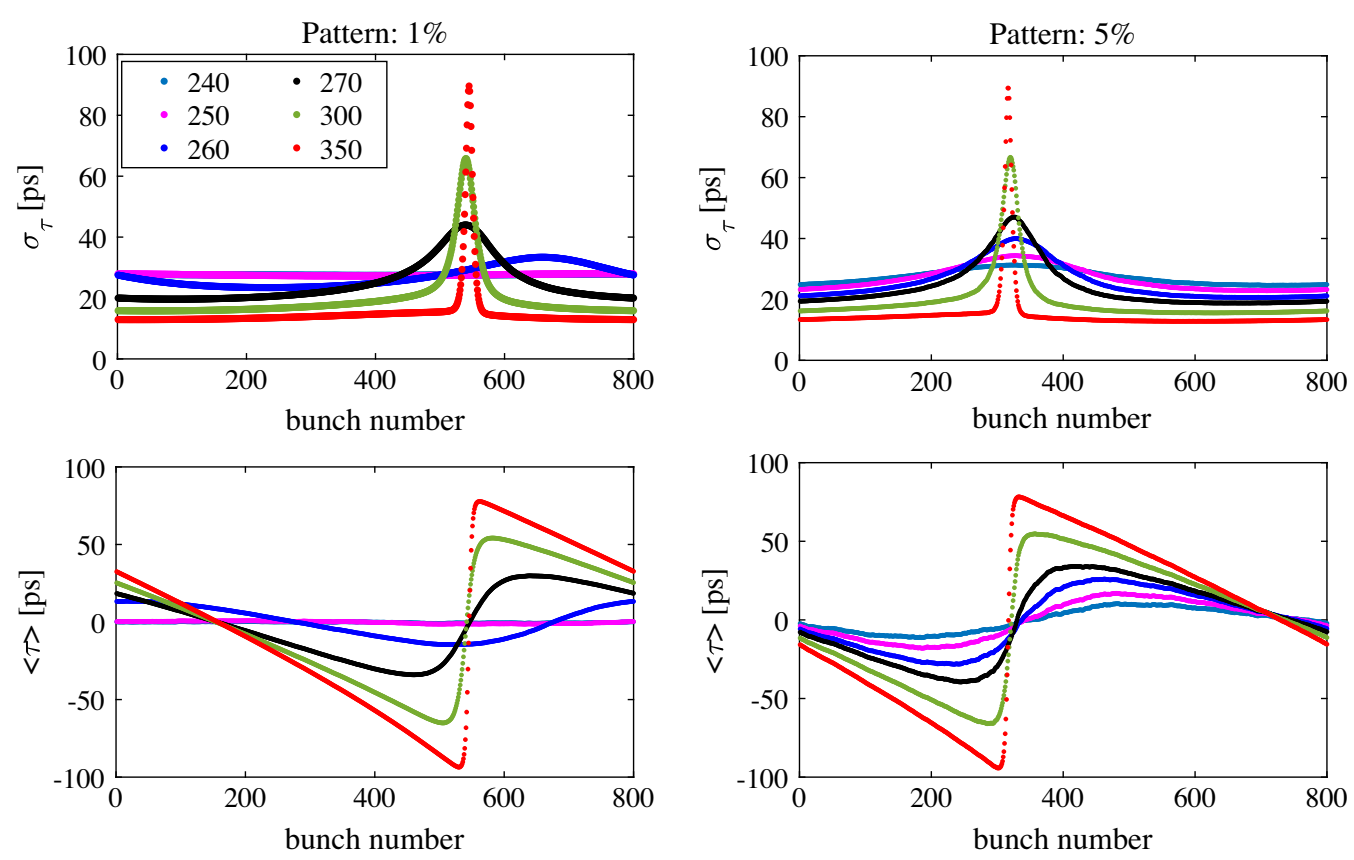

FIG. 13. RMS bunch lengths (top) and centroid positions (bottom) vs bunch number, obtained by the semianalytical method. The legend gives the beam current in mA. Note that for the cases of 270, 300, and $350 \mathrm{~mA}$ with $1 \%$ charge error, the resulting distribution changes periodically with the number of iterations, while only the results of the final iteration are illustrated.

\section{B. Nonuniform charge configuration}

We consider the complete fill pattern with fraction charge error $\sigma_{q}$ of $1 \%, 2 \%$ and $5 \%$ respectively. The errors are imposed to the charges using a random Gaussian distribution with $3 \sigma_{q}$ cut off. We also studied these cases using the semianalytical method. The state of results for different errors and currents can be classified into two types, one is convergent and denoted by the symbol " $C$," the other is periodically varied with the number of iterations and denoted by the symbol $P$, as summarized in Table V. We can see that for the cases of small charge error of $1 \%$ and $2 \%$, the charge variation across bunches does increase the occurrence threshold of PTBL. However, for the case of large charge error of 5\%, the results are always convergent.

Figure 13 shows the results of rms lengths and centroid positions versus bunch number after a large number of iterations for the cases of $1 \%$ and $5 \%$ charge errors. It can be clearly seen that no matter whether there exists a periodic transient, as beam current increases, the centroid transient becomes more severe with some of the bunches having double-bump profiles, and the overall bunch lengthening becomes worse. By comparing the two cases of $1 \%$ and $5 \%$, it can be found that both their overall centroid transients are almost the same at 270, 300 and $350 \mathrm{~mA}$, respectively. Furthermore, these centroid transients are also very close to those of the cases of uniform fill and uniformly distributed gaps with small gap width. It indicates that the overall centroid transient cannot be mitigated by seeking an appropriate fill pattern when beam current is much beyond the PTBL threshold estimated with uniform fill pattern.

The difference in the charge of each bunch will inevitably lead to its unique motion, which makes the behavior of PTBL presented in Table $\mathrm{V}$ may be significantly different from those shown in Figs. 1 and 5. In order to figure that out, we studied the case of $1 \%$ charge error at $270 \mathrm{~mA}$ by using the tracking method with the damping time set to $6 \mathrm{~ms}$ (to shorten the oscillation period according to Sec. IV D). Figure 14 shows the variations of centroid

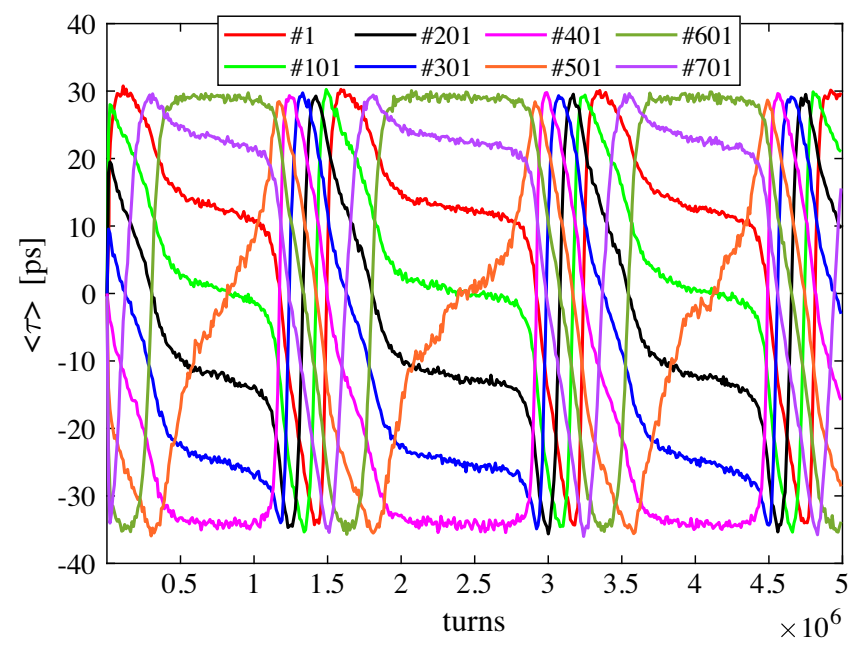

FIG. 14. Centroid position vs tracking turns for the case of $270 \mathrm{~mA}$ with $1 \%$ Gaussian charge error. The legend gives the bunch number. 
positions of eight representative bunches with tracking turns. It can be seen that the oscillation period of each bunch is identical as well as the oscillation amplitude, but the oscillation speed of each bunch is much different from each other and varies significantly with the tracking turn, which is obviously different from those shown in Figs. 1 and 5. It indicates that the charge variation across bunches spread their instantaneous oscillation speed.

\section{CONCLUSIONS}

In this paper, we have studied the characteristics of periodic transient beam loading for the HALF storage ring using both tracking simulation and semianalytical calculation. The PTBL effect happens when the current exceeds a threshold and a heavy PTBL can contribute to a poor bunch lengthening performance. The $R / Q$ and detuning frequency of $\mathrm{HC}$ and the product of momentum compaction factor $\left(\alpha_{c}\right)$ and the square of energy spread $\left(\sigma_{\delta}^{2}\right)$ play an important role in determining the threshold. Increasing the detuning frequency of $\mathrm{HC}$ away from the nearoptimum bunch lengthening condition is one way to avoid PTBL but it will cause much less bunch lengthening. A large $\alpha_{c} \sigma_{\delta}^{2}$ is also very helpful to increase the PTBL threshold, but its value is also limited since HALF adopts a multibend achromat lattice to reach a low emittance below $100 \mathrm{pm}$ rad. For a DLSR with low or medium energy, such as HALF, PTBL occurs at a low threshold of $R / Q \times I_{0}$. When a high beam current $I_{0}$ is required, the $R / Q$ of $\mathrm{HC}$ should be reduced to a certain value in order to avoid PTBL and obtain a desirable factor of bunch lengthening.

The existence of short-range wake can also increase the threshold if it does not cause a considerable energy spread growth. In the potential well distortion regime before the microwave instability happens, the damping time has no effect on the PTBL threshold. When the microwave instability happens, the semianalytical method becomes invalid, there will be a complex coupling interaction between the fundamental mode of $\mathrm{HC}$ and the short-range wake, which will even reduce the threshold. This coupling can be generally mitigated by reducing the radiation damping time. For the uniform fill pattern, the MC beam loading does not change the threshold, but will affect the centroid oscillation when beyond the threshold, that is, increasing the amplitude and reducing the frequency. For nonuniform but close to uniform fill pattern, the periodicity may be interrupted with a large gap width or charge error, but the overall bunch lengthening performance is not improved.

Finally, it should be noted that all calculations in this paper are based on the parameters of superconducting HC. Therefore, some conclusions mentioned above might not be applied to the cases of using normal conducting HC with low quality factor and high $R / Q$, which should be paid attention to and further investigated. In addition, we did not consider the influence of higher order modes in cavities, general fill pattern, and the coupling among multiple factors on PTBL. Further study will be performed in the future.

\section{ACKNOWLEDGMENTS}

We would like to thank our PRAB reviewers who made many important suggestions which helped us improve this paper. This work was supported by National Natural Science Foundation of China (No. 12105284 and No. 11875259) and the Fundamental Research Funds for the Central Universities (No. WK2310000090).

[1] Z. H. Bai, G. W. Liu, T. L. He, T. Zhang, W. W. Li, P. H. Yang, Z. L. Ren, S. C. Zhang, W. M. Li, G. Y. Feng, and L. Wang, A modified hybrid 6BA lattice for the HALF storage ring, in Proceedings of the IPAC2021, Campinas, SP (JACoW, Campinas, SP, Brazil, 2021), pp. 407-409, MOPAB112.

[2] M. Pedrozzi, J.-Y. Raguin, W. Gloor, A. Anghel, M. Svandrlik, G. Penco, P. Craievich, A. Fabris, C. Pasotti, E. Chiaveri, R. Losito, S. Marque, O. Aberle, P. Marchand, P. Bosland, S. Chel, P. Brédy, and G. Devanz, SLS Operational Performance with Third Harmonic Superconducting System, in Proceedings of the 11th Workshop RF Superconductivity, Lübeck, Germany (JACoW, Lübeck, Germany, 2003), pp. 91-94, MOP25

[3] T. L. He, Z. H. Bai, W. W. Li, G. W. Liu, H. L. Xu, S. C. Zhang, G. Y. Feng, L. Wang, and W. M. Li, Bunch lengthening of the HALF storage ring in the presence of passive harmonic cavities, in Proceedings of the IPAC2021, Campinas, SP (JACoW, Campinas, SP, Brazil, 2021), pp. 2082-2085, TUPAB265.

[4] T. L. He, Z. H. Bai, W. W. Li, G. W. Liu, H. L. Xu, S. C. Zhang, G. Y. Feng, L. Wang, and W. M. Li, Periodic transient beam loading effects predicted by a semianalytical method, in Proceedings of the IPAC2021, Campinas, SP (JACoW, Campinas, SP, Brazil, 2021), pp. 2086-2088, TUPAB266.

[5] T. He and Z. Bai, Graphics-processing-unit-accelerated simulation for longitudinal beam dynamics of arbitrary bunch trains in electron storage rings, Phys. Rev. Accel. Beams 24, 104401 (2021).

[6] J. M. Byrd, S. De Santis, J. Jacob, and V. Serriere, Transient beam loading effects in harmonic rf systems for light sources, Phys. Rev. ST Accel. Beams 5, 092001 (2002).

[7] N. Yamamoto, T. Takahashi, and S. Sakanaka, Reduction and compensation of the transient beam loading effect in a double rf system of synchrotron light sources, Phys. Rev. Accel. Beams 21, 012001 (2018).

[8] A. Gamelin, Harmonic cavities studies and applications for SOLEIL upgrade: bunch lengthening, transient beam loading and instabilities, 8th Low Emittance Rings Workshop (2020), https://agenda.infn.it/event/20813/ contributions/110228/attachments/76426/98245/ LER2020_HC_studies.pdf. 
[9] S. Y. Lee, Accelerator Physics (World Scientific Publishing, Singapore, 2004), pp. 136-137.

[10] R. A. Bosch, K. J. Kleman, and J. J. Bisognano, Robinson instabilities with a higher-harmonic cavity, Phys. Rev. ST Accel. Beams 4, 044401 (2021).

[11] M. Venturini, Passive higher-harmonic rf cavities with general settings and multibunch instabilities in electron storage rings, Phys. Rev. Accel. Beams 21, 114404 (2018).

[12] Å. Andersson, J. Breunlin, F. Cullinan, D. McGinnis, S. Molloy, D. Olsson, M. Sjöström, and P. F. Tavares, Feedbacks against Collective Instabilities at MAX IV, Joint ARIES workshop on Electron and Hadron Synchrotrons: Next Generation Beam Position Acquisition and Feedback Systems, Barcelona (2018), https://indico.cern.ch/event/ 743699/contributions/3112143/attachments/1750504/ 2840093/Cullinan_ARIESfbwkshpNov18.pdf.

[13] T. He, W. Li, Z. Bai, and L. Wang, Longitudinal equilibrium density distribution of arbitrary filled bunches in presence of a passive harmonic cavity and the short range wakefield, Phys. Rev. Accel. Beams 24, 044401 (2021).

[14] https://github.com/hetianlong-afk/BunchLengthening.

[15] T. Olsson, F. J. Cullinan, and $\AA$. Andersson, Self-consistent calculation of transient beam loading in electron storage rings with passive harmonic cavity, Phys. Rev. Accel. Beams 21, 120701 (2018).

[16] V. Smaluk, Y. J. Li, Y. Hidaka, T. Tanabe, O. Chubar, L. Wiegart, A. Blednykh, B. Bacha, and T. Shaftan, Effect of undulators on magnet lattice and emittance, Phys. Rev. Accel. Beams 22, 124001 (2019).

[17] H. Feng, S. De Santis, K. Baptiste, W. Huang, C. Tang, and D. Li, Proposed design and optimization of a higher harmonic cavity for ALS-U, Rev. Sci. Instrum. 91, 014712 (2020).

[18] P. F. Tavares, ̊. Andersson, A. Hansson, and J. Breunlin, Equilibrium bunch density distribution with passive harmonic cavities in a storage ring, Phys. Rev. ST Accel. Beams 17, 064401 (2014).

[19] K. Y. Ng, Physics of Intensity Dependent Beam Instabilities (World Scientific Publishing, Singapore, 2006), pp. 58-61.

[20] A. W. Chao, Physics of Collective Beam Instabilities in High Energy Accelerators (John Wiley \& Sons, New York, 1993), pp. 279-282.

[21] R. Nagaoka and K. L. F. Bane, Collective effects in a diffraction-limited storage ring, J. Synchrotron Radiat. 21, 937 (2014). 